\title{
Staphylococcal toxic shock syndrome with pre- exposure to COX-2 inhibitor: Symptom masking and delayed treatment
}

\author{
Min Wei Ching ${ }^{1}$ and Chee Ping Chong ${ }^{2^{*}}$ \\ ${ }^{1}$ Pharmacy Department, Putrajaya Hospital, Federal Territory of Putrajaya, Malaysia \\ ${ }^{2}$ Discipline of Clinical Pharmacy, School of Pharmaceutical Sciences, Universiti Sains Malaysia (USM), Penang, \\ Malaysia
}

\begin{abstract}
Due to uncommon occurrence of staphylococcal toxic shock syndrome (TSS) in male patient, streptococcal origin is often suspected and treated as such. Pre-exposure to nonsteroidal anti-inflammatory drugs had been associated with masking of inflammatory symptoms. These two factors may lead to delay in receiving appropriate antibiotic treatment. We present a case of staphylococcal toxic shock in a diabetic male. He was initially presented to emergency department with abdominal pain, leg swelling and shortness of breath. The patient was having minor skin ulcerations twelve days before admission. He was prescribed with etoricoxib for his shoulder pain for 3 days prior to admission. Initial presentations upon admission showed upper gastrointestinal bleeding, metabolic acidosis and acute kidney injury. He developed TSS with multiorgan failure one day later. Initial diagnosis was streptococcal TSS and therefore intravenous immunoglobulin and clindamycin was initiated. Nevertheless, repeated culture from blood and wound showed staphylococcal infection. The diagnosis was changed to staphylococcal TSS and intravenous cloxacillin was initiated. However, the patient succumbed to the illness after 11 days of admission. In this case report, pre-exposure to etoricoxib may cause masking of inflammatory symptoms and rapid progression of a wound infection into TSS. Rarity of staphylococcal TSS in male and difficulties in differentiating between streptococcal and staphylococcal TSS led to delay in appropriate treatment.
\end{abstract}

Key Words: Toxic shock syndrome, staphylococcal, COX-2 inhibitor, etoricoxib

\section{Introduction}

Staphylococcal toxic shock syndrome (TSS) is commonly reported among menstruating women related to the use of tampons (1). In recent years, the proportion of non-menstruating staphylococcal TSS cases has been increasing (2) and are more deadly than menstruating cases (3). The mortality of non-menstruating staphylococcal TSS was reported to be $22 \%$ (1). Nevertheless, most cases involved women, children and adolescents. Male involvement had been uncommonly reported.

Nonsteroidal anti-inflammatory drugs (NSAIDs) had been implicated in severe sepsis and septic shock $(4,5)$. It had been suggested that prior NSAIDs use could mask the inflammatory symptoms and thus delay antibiotic treatment. There are also reports that suggested NSAIDs predispose patient to septic shock and enhance progression of skin and soft tissue infection.
However, the relationship between NSAIDs and development of staphylococcal TSS remains inconclusive. Here, we present a case of staphylococcal TSS in a 59 years old male with pre-exposure to COX-2 inhibitor.

\section{Case report}

A 59 years old male was presented to the emergency department with abdominal pain, bilateral leg swelling and shortness of breath. He complained of leg pain after walking down 18 floors of condominium two days ago and was relatively well the day before hospital admission. Twelve days before the admission, he was involved in an occupational acid splash which resulting in minor skin ulcerations. The ulcerations were managed by corticosteroid cream and aqueous cream. Also, etoricoxib $90 \mathrm{mg}$ daily and gabapentin $300 \mathrm{mg}$ daily were prescribed for his shoulder pain for the past 3 days. His past 
medical history included hypertension, diabetes and dilated cardiomyopathy with non-compliant to medications. The patient had no history of infection or antibiotic consumption for the past one month.

At the emergency department, he became hypotensive (blood pressure: $60-70 / 30-50 \mathrm{mmHg}$ ) and collapsed. Inotropes were initiated and he was admitted to intensive care unit and required mechanical ventilation. Initial examinations revealed a low grade fever, tachycardia, bilateral crepts on lower zones of the lung, distended abdomen and coffee ground aspirate from Ryle's tube. Laboratory findings showed an initially low blood $\mathrm{pH}$ (7.17) which normalized after one week and a high creatine phosphokinase level $(3160$ $\mathrm{U} / \mathrm{L})$. White blood cell was low on admission $\left(3.2 \times 10^{3} / \mu \mathrm{L}\right)$ but showed improvement over one week before started to decrease to $1.7 \times 10^{3} / \mu \mathrm{L}$ on Day 11. Hemoglobin value was decreased over his hospitalization from $14.7 \mathrm{~g} / \mathrm{dL}$ on admission to $9.8 \mathrm{~g} / \mathrm{dL}$ on Day 11. Platelet count exhibited the same pattern of decrease from $129 \times 10^{3} / \mu \mathrm{L}$ on admission to $35 \times 10^{3} / \mu \mathrm{L}$ after 10 days. The patient had high prothrombin time (16.9 to 28.0 seconds) and low calcium level $(>2.0 \mathrm{mmol} / \mathrm{L})$ throughout his hospitalization. The laboratory parameters of the patient are shown in Table 1.

The patient was initially diagnosed with acute kidney injury with creatinine clearance less than 15 $\mathrm{ml} / \mathrm{min}$, upper gastrointestinal bleeding secondary to NSAIDs and rhabdomyolysis. Continuous venovenous hemofiltration was initiated to manage the acute kidney injury. On day 2, he developed bluish discoloration with multiple blisters and lesions over his right calf and thigh, bilateral leg edema and macular erythroderma. He was then diagnosed with TSS due to streptococcus with multiorgan failure and necrotizing fasciitis. Imipenem, clindamycin and doxycycline were initiated for broad-spectrum coverage included streptococcal and vibrio vulnificus infection. However, the limb lesions worsened into multiple bullae and vesicles, requiring wound debridement. Necrotizing fasciitis was then ruled out on day 4 as wound debridement revealed healthy fascia and muscle. Intravenous immunoglobulin was initiated. Blood and tracheal aspirate revealed methicillin-sensitive Staphylococcal aureus (MSSA) on day 4. Both chest X-ray and ultrasound abdomen findings were normal.

The right lower limb lesions were later progressed to left limb and both arms with total involvement of $33 \%$ of body surface area. Repeated blood culture reported MSSA sensitive to cloxacillin. The diagnosis was then changed to septic shock secondary to staphylococcal TSS and intravenous cloxacillin was started. Despite improvement in the lesions in the extremities, the patient succumbed to the illness on day 11 of admission.

\section{Discussion}

TSS is a severe diseases characterized by rapidity of clinical manifestations and progressions by toxin-producing strains of Staphylococcus aureus and Streptococcal pyogene (1). Although staphylococcal TSS is often associated with menstruating women, non-menstruation staphylococcal should receive equal awareness given by its higher mortality rate (3). Non-menstruating staphylococcal TSS may result from disruption of the skin or mucous

Table 1. Body temperatures, renal and liver profile of the patient

\begin{tabular}{lcccccccc}
\hline Characteristic/ Day & 1 & 3 & 5 & 7 & 8 & 9 & 10 & 11 \\
\hline Body Temperature $\left({ }^{\circ} \mathrm{C}\right)$ & 37.3 & 39.0 & 37.0 & 39.5 & 37.6 & 37.0 & 36.5 & 36.5 \\
$\quad$ Full blood count & & & & & & & & \\
$\quad$ White blood cell $\left(\mathrm{x} 10^{3} / \mu \mathrm{L}\right)$ & 3.2 & 3.4 & 5.8 & 11.2 & 10.3 & 6.4 & 5.1 & 1.7 \\
$\quad$ Platelet $\left(\mathrm{x} 10^{3} / \mu \mathrm{L}\right)$ & 129 & 59 & 15 & 22 & 23 & 29 & 29 & 35 \\
Renal profile & & & & & & & & \\
$\quad$ Urea $(\mathrm{mmol} / \mathrm{L})$ & 22.9 & 17.0 & 13.9 & 24.0 & 29.2 & 31.6 & 30.8 & 30.5 \\
$\quad$ Serum creatinine $(\mu \mathrm{mol} / \mathrm{L})$ & 423 & 199 & 131 & 200 & 227 & 224 & 213 & 211 \\
Liver function test & & & & & & & & \\
$\quad$ Albumin $(\mathrm{g} / \mathrm{L})$ & 17 & 17 & 14 & 12 & 14 & 13 & - & - \\
$\quad$ Total bilirubin $(\mu \mathrm{mol} / \mathrm{L})$ & 17 & 27 & 70 & 109 & 70 & 146 & - & - \\
$\quad$ Alanine aminotransferase $(\mathrm{U} / \mathrm{L})$ & 90 & 161 & 128 & 67 & 128 & 16 & - & - \\
$\quad$ Aspartate aminotransferase $(\mathrm{U} / \mathrm{L})$ & 163 & 105 & 148 & 143 & 148 & 105 & - & - \\
$\quad$ Creatine phosphokinase $(\mathrm{U} / \mathrm{L})$ & 3160 & - & - & - & - & - & - & - \\
\hline
\end{tabular}


membranes such as trauma, burns or after surgical procedure (1). During history taking, the patient denied any history of infection or antibiotic usage for the past one month. Thus, the skin ulcerations occurred before admission or unnoticed wound due to diabetic neuropathy might be the source of infection in this case.

Staphylococcal TSS shared similar clinical presentation with streptococcal TSS (Table 2). Salient features of staphylococcal TSS which occurred in this patient includes fever more than $38.9^{\circ} \mathrm{C}$, hypotension, desquamation and multisystem involvement such as severe myalgia, elevated creatine phosphokinase, renal impairment, total bilirubin raised twice-upper limit of normal and platelet count less than $100 \times 10^{3} /$ $\mu \mathrm{L}$ (1). Some overlapping manifestations with streptococcal TSS such as skin manifestation, hypotension, respiratory distress, renal and liver impairment and coagulopathy may leads to late diagnosis of this disease. Nevertheless, literatures had reported that desquamation is characteristic late presentation in staphylococcal TSS which is 10 to 21 days after onset of illness (1). Besides, multiorgan failure due to staphylococcal TSS occurs slightly later in disease development if compared to streptococcal TSS (3). However, as streptococcus is more commonly associated with
TSS, it is inevitable that the patient in this case was highly suspected of streptococcal infection rather than staphylococcal infection. This is due to the fact that the patient is a male and necrotizing fasciitis is suspected.

Previous case reports had revealed the possible relationship between the use of NSAIDs with the development of toxic gram positive infections $(4,5)$. However, most studies involved streptococcal infection and rarely staphylococcal infection $(6,7)$. Gonzalez et al. (4) had reported 14 cases of severe sepsis in adolescents due to Staphylococcus aureus in which 11 cases involved the consumption of NSAIDs. The risk of mortality was higher in the 11 cases of the NSAIDs group as compared to the 3 cases which did not involve NSAIDs usage. Among these 11 cases, 8 cases involved the consumption of NSAIDs before admission. Nevertheless, the exact time of NSAIDs consumption were only documented among 2 out of these 8 patients, which were approximately 5 and 7 days respectively before admission $(4,8)$. Ibuprofen was most commonly used among the patients, followed by ketorolac and naproxen (4). In our case, the patient consumed etoricoxib, a COX-2 inhibitor at 3 days before the admission.

Table 2. Clinical presentation of staphylococcal and streptococcal TSS (adapted from Lappin and Ferguson, 2009)

\section{Staphylococcal TSS}

1. Fever $\geq 38.9^{\circ} \mathrm{C}$

2. Rash - diffuse macular erythroderma

3. Desquamation -1-2 weeks after onset of illness, especially of palms and soles

4. Hypotension - systolic blood pressure $\leq 90$ $\mathrm{mmHg}$

5. Multi-system involvement -3 or more of the following:
a) Gastrointestinal - vomiting or diarrhea
b) Muscular - severe myalgia or elevated creatine phosphokinase
c) Mucous membranes - vaginal, oropharyngeal, conjunctival hyperemia
d) Renal - blood urea nitrogen or creatinine twice upper limit of normal
e) Hepatic - total bilirubin twice upper limit of normal
f) Hematological - platelets $\leq 100 \times 10^{3} / \mu \mathrm{L}$
g) Central nervous system - disorientation or alterations in consciousness without focal neurological signs

\section{Streptococcal TSS}

1. Hypotension - systolic blood pressure $\leq 90$ $\mathrm{mmHg}$

2. Two or more of the following signs:

a) Renal impairment - creatinine $>177$ $\mu \mathrm{mol} / \mathrm{L}(>2 \mathrm{mg} / \mathrm{dL})$

b) Coagulopathy - platelets $\leq 100 \times 10^{3} / \mu \mathrm{L}$ or disseminated intravascular coagulation

c) Hepatic involvement - alanine aminotransferase, aspartate aminotransferase or total bilirubin twice upper limit of normal

d) Adult respiratory distress syndrome

e) Generalized, erythematous, macular rash that may desquamate

f) Soft-tissue necrosis, including necrotizing fasciitis or gangrene 
NSAIDs exert its anti-inflammatory effects via suppression of granulocyte functions, chemotaxis, oxidative outburst, phagocytosis and bacterial killing. According to Stevens (5), pre-treatment with NSAIDs enhances production of tumor necrosis factor (TNF), which leads to higher levels of circulating TNF, one of the major mediators of shock and organ failure. NSAIDs inhibit conversion of arachidonic acid to prostaglandin $\mathrm{E}_{2}$ $\left(\mathrm{PGE}_{2}\right)$ in the brain and periphery. $\mathrm{PGE}_{2}$ is a potent feedback inhibitor of monocyte synthesis of TNF, and in its absence, unregulated TNF synthesis occurs. NSAIDs also inhibit leukocytemediated host defense mechanisms, suppress fever and enhance cytokine production by host cells. This causes masking of clinical signs and symptoms of infection while enhancing overproduction of cytokine. Therefore, NSAIDs use not only could delay appropriate diagnosis and antibiotic treatment, it could also facilitate local spread of infection and thus, predispose patients to shock and organ failure (5). The drastic development of severe sepsis and shock in the present case may explain by the use of NSAIDs therapy.

The relationship between COX-2 inhibitor and staphylococcal infection of skin and soft tissue was demonstrated by an in vitro study by Bernard and Gallo (9). Normal human epidermal keratinocytes (NHEK) in the skin plays a role in the bactericidal activity in skin and soft tissue infection via initiating the release of cytokines and chemokines that stimulate and recruit natural killer cells and $\mathrm{T}$ lymphocytes to the site of infection. Activation of NHEK induces COX-2 to increase production of antimicrobial peptides (AMPs) which are responsible to kill pathogens and stimulate release of cytokines and chemokines, recruit other immune cells and acting as adjuvant for antibody production. One of the AMPs, human $\beta$-defensin-3 (hBD3) is a critical determination of anti-staphylococcal activity of keratinocytes. COX-2 inhibition attenuates the production of AMPs, including $\mathrm{hBD} 3$. This reduces anti-staphylococcal activity of NHEK and thereby adversely affects the outcome of skin and soft tissue infections (9).

Case-control study by Legras et al. (10) demonstrated that NSAIDs use significantly delay antibiotic prescription. However, the study did not find any correlation that NSAIDs exposure during evolving bacterial infection is associated with an increased risk for severe sepsis of septic shock. This could be due to low incidence of skin and soft tissue infection (11\%) included into the study and high rate of undocumented infection in the control group resulted from unavailable microbiological documentation (10). Nevertheless, the role of NSAID predisposes patient to TSS remains argumentative due to lack of conclusive literature.

In the present case, the NSAID may have masked the inflammatory symptoms and delayed the patient from seeking appropriate treatment. According to Chesney (11), staphylococcal TSS has rapid onset and manifestation due to short incubation period. It causes rapid onset of hypotension, precipitated by decrease in vasomotor tone and rapid leakage of fluid from intravascular to the interstitial space. This causes pooling of blood into the peripheral vasculature, and thus manifested as extensive generalized peripheral edema. In our case, the patient was presented with bilateral edema and shortness of breath. He became hypotensive and developed shock in a very short period of time. Multiorgan failure developed shortly after. Recent studies have reported on the possible role of COXinhibitor in ameliorating the destructive pathophysiology and modulating immune system to combat severe sepsis $(12,13)$. However, most studies were performed using streptococcus strain rather than staphylococcus. Therefore, further studies are required to explore the possibilities of COX-inhibitor in severe sepsis.

In summary, to our knowledge this is the first report of staphylococcal TSS in a middle age male pre-exposed to COX-2 inhibitor. Lack of exposure to staphylococcal TSS especially in nonmenstruating male patient causes diagnosis difficulties and thus delays appropriate antibiotic treatment. Although the causal-relationship of NSAIDs in staphylococcal TSS had yet to be determined, its plausibility of masking the inflammatory symptoms could not be rule out. Clinicians should be cautious about the use of COX-2 inhibitor among the patients which are at risk of sepsis.

\section{References}

1. Lappin E, Ferguson AJ. Gram-positive TSSs. Lancet Infect Dis 2009; 9: 281-290.

2. Hajjeh RA, Reingold A, Weil A, et al. TSS in the United States: surveillance update, 1979-1996. Emerg Infect Dis 1999; 5: 807-810.

3. Vincent JM. Staphylococcal and streptococcal TSSs; in: Yamamoto LG, Inaba AS, Okamoto JK, Patrinos ME, Yamashiroya VK (eds): Case based pediatrics for medical students and residents. 
Honolulu, Department of Pediatrics, University of Hawaii John A, Burns School of Medicine, 2004, pp 218-222.

4. Gonzalez BE, Hulten KG, Hammerman WA, et al. Nonsteroidal anti-inflammatory drugs and invasive staphylococcal infections: the cart or the horse?: In reply. Pediatrics 2005; 115: 1791.

5. Stevens DL. Could nonsteroidal antiinflammatory drugs (NSAIDs) enhance the progression of bacterial infections to TSS? Clin Infect Dis 1995; 21: 977-980.

6. Hamilton SM, Bayer CR, Stevens DL, Lieber RL, Bryant AE. Muscle injury, vimentin expression, and nonsteroidal anti-inflammatory drugs predispose to cryptic group A streptococcal necrotizing infection. J Infect Dis 2008; 198: 1692-1698.

7. Mulla ZD. Nonsteroidal anti-inflammatory drugs and hypotension among patients hospitalized for invasive Group A streptococcal disease. Ann Epidemiol 2003; 13: 543-544.

8. Gonzalez BE, Martinez-Aguilar G, Hulten KG, et al. Severe staphylococcal sepsis in adolescents in the era of community-acquired methicillinresistant Staphylococcus aureus. Pediatrics 2005; 115: 642-648.

9. Bernard JJ, Gallo RL. Cyclooxygenase-2 enhances antimicrobial peptide expression and killing of Staphylococcus aureus. J Immunol 2010; 185: 6535-6544.

10. Legras A, Giraudeau B, Jonville-Bera AP, et al. A multicentre case-control study of nonsteroidal anti-inflammatory drugs as a risk factor for severe sepsis and septic shock. Crit Care 2009; 13:R43, DOI:10.1186/cc7766.

11. Chesney PJ. Clinical aspects and spectrum of illness of toxic shock syndrome: overview. Reviews of Infectious Diseases 1989; 11: 1-7.

12. Goldmann O, Hertzén E, Hecht A, et al. Inducible cyclooxygenase released prostaglandin $\mathrm{E}_{2}$ modulates the severity of infection caused by Streptococcus pyogenes. J Immunol 2010; 185: 23722381.

13. Aronoff DM. Cyclooxygenase inhibition in sepsis: is there life after death? Mediators of Inflammation 2012; Doi:10.1155/2012/696897. 